\title{
Sonication of porphyrin-nanotube composites: a cautionary tale
}

\author{
E. M. Ní Mhuircheartaigh ${ }^{1}$, W. J. Blau ${ }^{1}$, M. Prato ${ }^{2}$ and S. Giordani ${ }^{* 2,3}$ \\ ${ }^{1}$ School of Physics, University of Dublin Trinity College, College Green, D2 Dublin, Ireland \\ 2 Dipartimento di Scienze Farmaceutiche, Universita' degli Studi di Trieste, P.le Europa 1, 34127 Trieste, \\ Italy \\ ${ }^{3}$ School of Chemistry, trinity College Dublin, Dublin 2, Ireland \\ Received $z z z$, revised $z z z$, accepted $z z z$ \\ Published online $z x z$
}

PACS 61.46.Fg, 68.37.Ps, 78.67.Ch 81.07.De

\begin{abstract}
We report the spectroscopic changes of tetraphenylporphyrin carbon nanotube composites in a variety of chlorinated solvents such as chloroform, dichloroethane and dichlorobenzene as a result of sonication. Detailed data analysis suggests that protonation of the porphyrin core nitrogen atoms occurs as a result of sonodegradation of the solvent molecules. Extreme care must be taken when working with carbon nanotube composite solutions in chlorinated solvents, as sonication is always employed to disperse the nanotubes. This finding is of enormous relevance to the materials chemistry community since these solvents are widely used in preparation of carbon nanotube composite solutions. When working with molecules that are capable of acting as a base some amount of protonation is inevitable and this may dramatically change the physicochemical properties of the composite materials.
\end{abstract}

Copyright line will be provided by the publisher

\section{Introduction}

Functionalization of nanotubes is extremely important as it couples the mechanical and structural strength of the carbon skeleton with desirable optical properties imparted by the molecules that are used to decorate the carbon exo-surface. Porphyrins represent one such family of functional molecules with potential applications as sensitizers for photoactive switches, light-harvesting systems, in cancer photodynamic therapy and electronics. Porphyrins are also particularly important as suitable partners of carbon nanotubes in composites [1-8] due to their (electro) luminescence and photovoltaic properties as well as to their biocompatibility. Regardless of the functional molecule, sonication is a key step in the dispersion of single-walled carbon nanotubes when making composite solutions, and chloroform is frequently the solvent of choice. [9,10] However, sonication of a variety of halogenated solvents $[11,12]$ may produce degradation products that can react with moieties on the composite thus hampering and complicating both characterization and function of the resulting material. In the study reported herein, we employ absorption, emission and ${ }^{1} \mathrm{H}$-NMR spectroscopy to investigate the effect of sonication on the optical properties of tetraphenylporphyin solutions in chlorinated solvents before and after the addition of SWNT.

\section{Experimental Procedure}

Purified HiPCO single walled nanotubes (SWNT) were purchased from Carbon nanotechnologies Inc and used as supplied (lot \#PO289). Chemicals were purchased from commercial sources and were used as received. Chloroform was purified on a column of basic alumina (grade $\mathrm{I}, 10 \mathrm{~g} / 14 \mathrm{ml}$ ) in order to

* * Corresponding author: e-mail: giordans@tcd.ie Fax:+35316712826 
remove all impurities, including the residual traces of $\mathrm{HCl}$ always present in this commercially available organic solvent. SWNTs were added to porphyrin solutions such that the porphyrin : SWNT mass ratio was $1: 1$. Sonication was performed using a high power ultrasonic tip (model GEX600, 120W, 60kHz). Absorption and photoluminescence spectra were recorded with a Shimadzu UV3100 UV-Vis-NIR spectrometer and a Perkin-Elmer LS-55 luminescence spectrometer. Proton nuclear magnetic resonance $\left({ }^{1} \mathrm{H}-\mathrm{NMR}\right)$ spectra were recorded with a Bruker DPX 600 at $25^{\circ} \mathrm{C}$, using deuterated chloroform as lock.

\section{Results and Discussion}

The absorption spectrum of an unsonicated red solution of tetraphenylporphyin TPP (Fig. 1a left) in chloroform shows a typical Soret band at $418 \mathrm{~nm}$ and four Q-bands at 516, 550, 590 and $646 \mathrm{~nm}$. The absorption spectrum of the sonicated green TPP : SWNT composite solution (Fig. 1b, left) looks very different, with a Soret band at $446 \mathrm{~nm}$ and two Q-bands at 610 and $660 \mathrm{~nm}$.
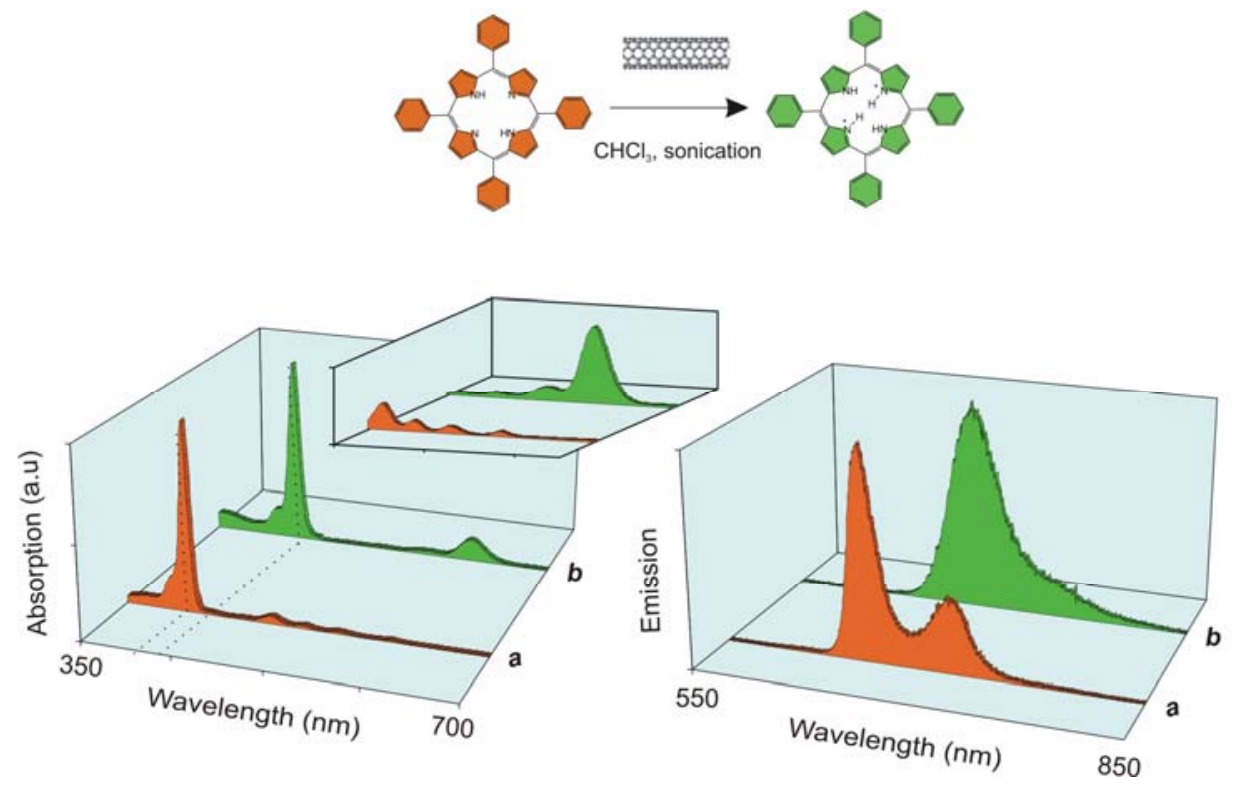

Fig. 1 Absorption (left) and emission (right) spectra of a solution of TPP (a) and of a composite solution of TPP : SWNTs sonicated for 1 minute (b) $\left(3 \mathrm{mg} / \mathrm{L}, \mathrm{CHCl}_{3}, 25^{\circ} \mathrm{C}\right)$.

Excitation at the Soret band of a chloroform solution of TPP results in two emission peaks at 650 and $715 \mathrm{~nm}$, typical for such porphyrin molecules (Fig. 1a, right). The photoluminescence spectrum of the TPP : SWNT composite solution is very different, showing a broad emission band with the maximum located at $685 \mathrm{~nm}$ (Fig. 1b, right).

We investigated the effect of sonication of chloroform on the porphyrin molecules alone. Upon sonication of a solution of TPP in chloroform the color of the solution changes from red to green. The Soret band is shifted to $446 \mathrm{~nm}$ and the characteristic porphyrin Q-bands are converted to two bands at 610 and $660 \mathrm{~nm}$. The photoluminescence spectrum shows one broad band at $685 \mathrm{~nm}$. The red shifted Soret band and the pattern of Q- bands in those sonicated solutions suggest that the porphyrin molecules exist in a protonated form. [13]. We investigated the effect of protonation on the spectroscopy of the porphyrins. Upon additition of hydrochloric acid to unsonicated porphyrin solution, the absorption and the emission spectra looks identical to those for the sonicated TPP and TPP : SWNT composite 
solutions. We propose that the hydrochloric acid created by sonodegradation of chloroform $[14,15]$ causes the protonation of the core nitrogen atoms of the porphyrins.

In order to confirm our hyphotesis we used proton nuclear magnetic resonance spectroscopy.

The ${ }^{1} \mathrm{H}-\mathrm{NMR}$ spectrum of a $\mathrm{CDCl}_{3}$ solution of TPP $\left(\mathrm{TPPH}_{2}\right.$ Fig. 2a) shows a singlet at $8.9 \mathrm{ppm}$ for the $\beta$ pyrrole protons $\mathrm{H}^{1}$, a doublet at $8.2 \mathrm{ppm}$ for the ortho-phenyl protons $\mathrm{H}^{2}$ and a multiplet at $7.8 \mathrm{ppm}$ corresponding to the overlapping signals for the metha-phenyl and the para-phenyl protons $\mathrm{H}^{3}$ and $\mathrm{H}^{4}$. The addition of acid alters significantly the chemical shifts of all the aromatic protons (Fig. 2c). The largest change is observed for the doublet of the ortho-phenyl protons, which shifts by ca. $+0.4 \mathrm{ppm}$ after the addition of an excess of trifluoroacetic acid, TFA $\left(\mathrm{H}^{6}\right)$. The metha and para-phenyl protons move downfield and broaden significantly $\left(\mathrm{H}^{7}, \mathrm{H}^{8}\right)$. The signal of the $\beta$-pyrrole protons moves upfield upon protonation $\left(\mathrm{H}^{5}\right)$. After two minutes sonication the 1H-NMR spectrum contain superimposed signals of both $\mathrm{TPPH}_{2}$ and $\mathrm{TPPH}_{4}{ }^{2+}$ at their normal chemical shift values (Fig. $2 \mathrm{~b}$ ). The ${ }^{1} \mathrm{H}-\mathrm{NMR}$ spectrum of a $\mathrm{CDCl}_{3}$ solution of the composite (Fig. 2d) contains signals of both $\mathrm{TPPH}_{2}$ and $\mathrm{TPPH}_{4}{ }^{2+}$. The signals are broadened, probably due to interactions of the porphyrin with the carbon nanotubes.

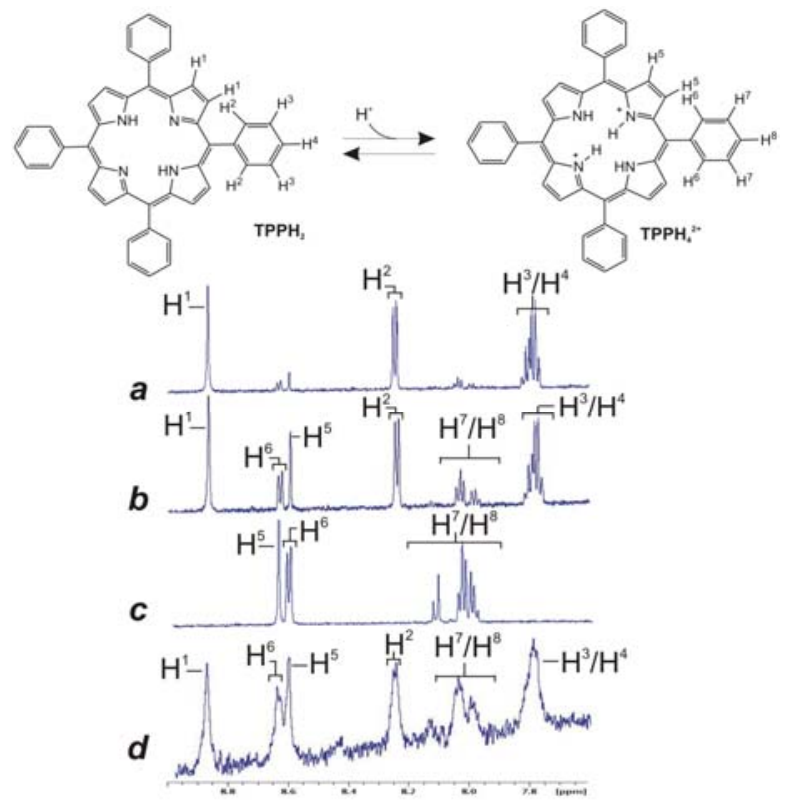

Fig. 2 Partial ${ }^{1} \mathrm{H}-\mathrm{NMR}$ spectra of a solution of TPP (a) before sonication, (b) after sonication for 2 minute (c) after addition of 10 equivalents of TFA and (d) of a composite solution of TPP: SWNTs $\left(3 \mathrm{mg} / \mathrm{L}, \mathrm{CDCl}_{3}, 25^{\circ} \mathrm{C}, 600\right.$ $\mathrm{KHz})$.

In order to explore if sonication of other solvents produce degradation products that can react with the porphyrin composites we performed sonication studies in various other solvents commonly used to disperse nanotubes. We observed the same spectral shifts upon sonication of TPP in dichloroethane (DCE) and dichlorobenzene (DCB). No spectral shifts were observed when dichloromethane (DCM), N,N-dimethylformamide (DMF), N-methylpyrrolidone (NMP) and tetrahydrofurane (THF) were used as solvents. Complete protonation of the porphyrins was found after sonication for 2 minutes and 30 seconds in the chlorinated solvents DCE and DCB respectively. No protonation was observed after up to 90 minutes sonication in DCM. This solvent was found to have a much lower sonochemical degradation rate compared to the other chlorinated solvents [10a]. Figure 3 illustrates the absorption spectra of TPP 
sonicated for one minute in DCM, DCE and DCB. It can clearly be seen that no protonation, partial and complete protonation were achieved respectively.

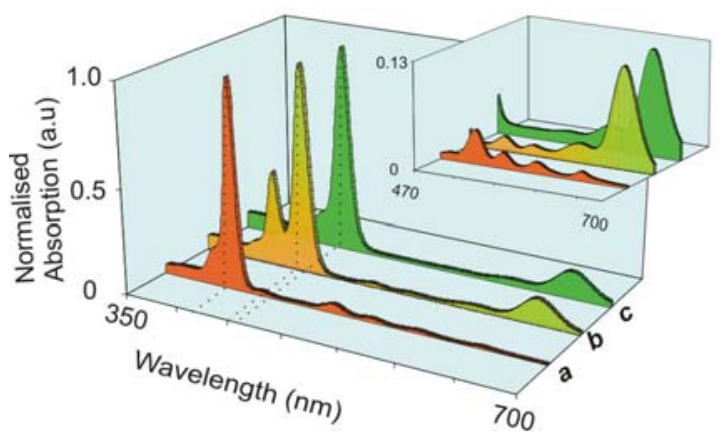

Fig. 3 Absorption spectra of a solution of TPP after sonication for 1 minute in (a) DCM, (b) DCE (c) DCB. (3mg/L, $\left.\mathrm{CHCl}_{3}, 25^{\circ} \mathrm{C}\right)$.

We demonstrated that spectroscopic changes of porphyrins in composite solutions are due to protonation of the porphyrin resulting from sonolysis of a variety of chlorinated solvents. TPP was protonated in chloroform, DCE and DCB upon sonication. No protonation was observed when DCM, DMF, NMP and THF were used as solvents.

Therefore, extreme care must be taken when working with carbon nanotube composite solutions in halogenated solvents as protonation of acid sensitive molecules may take place, changing their physicochemical properties.

Acknowledgements The authors wish to acknowledge the support of the European Union in the form of the European Community's Human Resources and Mobility Program under contract MERG-CT-2006-042036 [SMART NANOTUBES]. We also thank Science Foundation Ireland (SFI) and INSTM for financial support.

\section{References}

[1] H. Murakami, T. Nomura, N. Nakashima, Chem. Phys. Lett. 378, 481(2003).

[2] H. Li, B. Zhou, Y. Lin, L. Gu, W. Wang, K. A. S. Fernando, S. Kumar, L. F. Allard, Y. P. Sun, J. Am. Chem. Soc. 126, 1014 (2004).

[3] D. M. Guldi, H. Taieb, G. M. A. Rahman, N. Tagmatarchis, M. Prato, Adv. Mater, 17, 871(2005).

[4] J. Chen, C.P. Collier, J. Phys. Chem. B 109, 7605 (2005).

[5] H. Li, R. B. Martin, B. A. Harruff, R. A. Carino, L. F. Allard, Y. P. Sun, Adv. Mater. 16, 896 (2004).

[6] K. S. Chichak, A. Star, M. V. P. Altoe, J. F. Stoddart, Small 1, 452 (2005).

[7] M. Alvaro, P. Atienzar, P. de la Cruz, J. L. Delgado, V. Troiani, H. Garcia, F. Langa, A. Palkar, L. Echegoyen, J. Am. Chem. Soc. 128, 6626 (2006).

[8] F. Cheng, A. Adronov, Chem. Eur. J. 12, 5053 (2006).

[9] D. W. Steuerman, A. Star, R. Narizzano, H. Choi, R. S. Ries, C. Nicolini, J. F. Stoddart, J. R. Heath, J. Phys. Chem. B 106, 3124 (2002).

[10] A. Star, Y. Liu, K. Grant, L. Ridvan, J. F. Stoddart, D. W. Steuerman, M. R. Diehl, A. Boukai, J. R. Heath, Macromolecules 36, 553 (2003).

[11] A. J. Colussi, H. M. Hung, M. R. Hoffmann, J. Phys. Chem. A 103, 2696 (1999).

[12] A. Bhatnagart, H. Cheung, Environ. Sci. Techno. 28, 1481 (1994).

[13] J. Yu, B. S. Yu, K. Cheong, H. Choi, S. C. Yu, Bull. Korean Chem. Soc. 19, 439 (1998).

[14] H. Shemer, N. Narkis, Environ. Sci. Technol. 38, 4856 (2004).

[15] M. M. Castellanos, D. Reyman, P. Calle, J. Camacho, J. Ultrasonics Sonochemistry 5, 107 (1998). 